\title{
Sujeto Académico Neoliberal: Cuantificado, Digitalizado y Bibliometrificado
}

\section{Neoliberal Academic Subject: Quantified, Digitised and Bibliometrified}

\author{
Geo Saura * \\ Antonio Bolívar
}

Universidad de Granada, España

\begin{abstract}
Este artículo analiza, a partir de un enfoque de origen Foucaultiano, las formas en que el profesorado universitario actualmente se constituye a sí mismo, en lo que hemos dado en llamar "sujeto académico neoliberal". Es una primera exploración de una investigación más amplia. Desde esta mirada, analizamos los procesos de neoliberalización en el sujeto académico contemporáneo, mostrando cómo se configuran la formación y carrera universitaria de los jóvenes investigadores, dentro de la mercantilización dominante en la educación superior en España. Mediante 24 entrevistas semiestructuradas, de un trabajo más amplio de corte cualitativo, se interpretan los efectos de estas lógicas en el sujeto académico la Educación Superior en España. Creyendo que son libres en sus prácticas, mediante el invisible poder de unas formas blandas de gobernanza (soft governance), nuevas relaciones de conocimiento-poder (rankings, evaluaciones cuantificadoras) y procesos de digitalización (bibliometría, impactos) están provocando nuevas subjetividades académicas. Los análisis muestran cambios en los sistemas de cuantificación en la producción académica que provoca procesos de somatización del neoliberalismo, así como la configuración de nuevas subjetividades académicas a través de las digitalizaciones y el impacto bibliométrico.
\end{abstract}

Descriptores: Educación superior; Neoliberalismo; Gobernabilidad; Bibliometría; Digitalización.

This article analyses, from an approach of Foucaultian origin, the ways in which the university professors currently constitute themselves, in what we have called "neoliberal academic subject". It is a first exploration of a wider investigation. From this perspective, we analyse the processes of neoliberalization in the contemporary academic subject, showing how the formation and university career of young researchers are configured, within the dominant mercantilization in higher education in Spain. Through 24 semi-structured interviews, of a broader qualitative work, the effects of these logics are interpreted in the academic subject of Higher Education in Spain. Believing that they are free in their practices, through the invisible power of soft forms of governance (soft governance), new knowledge-power relations (rankings, quantifying evaluations) and digitalization processes (bibliometric, impacts) are causing new academic subjectivities. The analysis shows changes in the quantification systems in the academic production that causes processes of somatization of neoliberalism, as well as the configuration of new academic subjectivities through digitization and the bibliometric impact.

Keywords: Higher education; Neoliberalism; Governance; Bibliometrics; Digitalization.

*Contacto: geosaura@ugr.es

ISSN: $1696-4713$

Recibido: $\quad 21$ de abril 2019

www.rinace.net/reice/

$1^{\text {a }}$ Evaluación: 27 de junio 2019

revistas.uam.es/reice

$2^{\text {a }}$ Evaluación: 7 de julio 2019

Aceptado: $\quad 15$ de julio 2019 


\section{Introducción}

La capacidad del académico para ser uno mismo se ve limitada por mecanismos institucionalizados de poder y control que dirigen su acción y modifican su identidad profesional. Estas influencias, aceptadas y asimiladas como imperativos de la profesión, dirigen el desarrollo de la actividad profesional dando lugar a nuevas subjetividades neoliberales académicas. Los sujetos académicos actuales están cuantificados (Lupton, 2016), datificados (Cheney-Lippold, 2017), digitalizados (Lupton, Mewburn y Thomson, 2018; Moore y Robinson, 2016) y metrificados (Beer, 2016). La nueva gobernanza digital de la educación (Williamson, 2017) emplea la bibliometría, las cuantificaciones, las datificaciones y la digitalización como formas de control. Son tecnologías políticas contemporáneas que comienzan a conformar nuevas racionalidades de gobierno a través de relaciones de poder y control en la Educación Superior. Estas nuevas formas de gobernanza amplían, por un lado, los procesos de control regidos por el poder de la numeración (Badiou, 2008 Howie, 2005; Rose, 1999) y, por otro, las lógicas de la tecnología política de lo que Lyotard (1979) definió como performatividad o acciones desarrolladas por los sujetos con carácter finalista (relación input/output) (Barnett, 1996; Cowen, 1996).

Las nuevas tecnologías políticas han introducido lógicas basadas en la producción que están configurando al sujeto académico contemporáneo. Dichas tecnologías políticas, o racionalidades gubernamentales, se conforman siempre bajo una red de aparatos, técnicas y procedimientos de gobierno que, desde el exterior, actúan provocando la subjetivación del sujeto (Rose, 1999). De esta manera, el sujeto se comprende a través de la unificación de dos significados que se interponen entre sí: sujeto que se erige bajo procesos de sumisión a través del control y la dependencia respecto a otros/as; y sujeto atado a su identidad por la conciencia que tiene de sí (Foucault, 1988).

La falta de control del sujeto sobre sí mismo, o proceso de sujeción, es el resultado de las tecnologías políticas y racionalidades de gobierno que actúan sobre el yo. Una sujeción como "proceso de devenir subordinado al poder, así como el proceso de devenir sujeto" (Butler, 2010, p. 12). El sujeto como proyecto no es algo concluso, sino que siempre está constituyéndose a través de sujeciones; un devenir. El devenir del sujeto académico contemporáneo ya no requiere un control externo para su producción. La libertad ahora construye un devenir sujeto activo en la producción incesante.

A lo largo de este artículo, abordamos, en primer lugar, las dos corrientes de análisis del neoliberalismo en la Educación Superior, así como las prácticas asociadas a las lógicas de cambio en el profesorado universitario. Seguidamente, examinamos la política universitaria de la Educación Superior en España a través de los cambios en el sistema de acreditación de la calidad académica (sexenios y acreditaciones) y los procesos de mercantilización regidos por la industria de las publicaciones. Posteriormente, nos ocupamos de contextualizar la investigación en la Educación Superior en España a través del uso de entrevistas semiestructuradas. Los dos últimos apartados de resultados, mediante la interpretación y la reflexión, en una primera exploración, exponen los resultados extraídos de las entrevistas sobre los sistemas de cuantificación de la producción académica, los procesos de somatización del neoliberalismo, así como los cambios que los procesos de digitalización y la bibliometría están logrando en la configuración de nuevas subjetividades académicas. 


\section{Neoliberalismo en la Educación Superior}

En el estudio del neoliberalismo en la Educación Superior pueden distinguirse dos perspectivas diferenciadas. Una de ellas se fundamenta en los enfoques de condición estructural de las políticas y otra se centra en el análisis de las racionalidades neoliberales. De un modo similar, autores como Tight (2019) y Marginson (2013, p. 54) diferencian entre un discurso neoliberal centrado en la comercialización y la liberalización, asociadas a la estructura, y otro focalizado en los aspectos de la Nueva Gestión Pública o racionalidades neoliberales. Los rasgos de estas dos perspectivas de análisis se aclaran a continuación, siendo de utilidad para la comprensión de este texto.

Con respecto a la primera visión, durante las últimas dos décadas, después de lo que Slaughter y Leslie (2001) expandieron como capitalismo académico (Brunner et al., 2019), ha habido un conjunto de análisis que han demostrado los procesos estructurales de la globalización neoliberal en la Educación Superior. Dichos análisis se han centrado en comprender las transformaciones desde la economía política. Partiendo de esta mirada, y analizando "mecanismos externos" (Slaughter y Leslie, 2001, p. 155), se ha evidenciado cómo la Educación Superior ha vivido profundas transformaciones en la mayoría de países a causa de la globalización y la economía del conocimiento (Torres y Schugurensky, 2002). Las transformaciones políticas que componen estos mecanismos externos del neoliberalismo estructural (Ong, 2007; Springer, 2012) en las universidades, manifiestan la puesta en marcha de políticas de liberalización, mercantilización, comercialización, desregularización y privatización (Barnet, 2005; Slaughter y Leslie, 2001). La cultura de la auditoría sobre la producción de las universidades (Shore y Wright, 2015), las políticas de rankings, la competencia entre países y la comprensión de la universidad como capitalismo cognitivo (De Angelis y Harvie, 2009) forman parte también de estos cambios estructurales producidos por lógicas neoliberales durante las últimas dos décadas. En definitiva, las principales conclusiones de esta corriente de análisis muestran el desarrollo de políticas de mercado, la obtención de beneficios económicos de lo público, así como la entrada de actores privados para la gestión y el liderazgo del conocimiento en las universidades.

La segunda perspectiva, centrada en las racionalidades neoliberales, surge con la pretensión de traspasar las transformaciones estructurales de la Educación Superior, y lo hace utilizando la noción foucaultiana de gubernamentalidad para abordar las prácticas neoliberales en los individuos. Pone el foco de análisis en las estrategias de gubernamentalidad, o conducción de las conductas (Foucault, 1991), que se rigen mediante la extensión de nuevas racionalidades de gobierno (Foucault, 2007) que dirigen las acciones de los sujetos mediante la libertad y la competencia (Ong, 2007; Rose, 1999). Bajo esta perspectiva, desde la última década, se ha evidenciado cómo se han ejercido procesos de gubernamentalidad neoliberal en la Educación Superior mediante políticas de auto-gobierno que fomentan la individualidad y las prácticas de rendición de cuentas (Cannizzo, 2015; Jankowski y Provezis, 2014; Morrissey, 2013; Ozga, Seddon y Popkewitz, 2006). Otra de las claves de estos análisis ha sido poner la mirada en los procesos de gobierno de los sujetos a través de los discursos de "emprendimiento y competitividad" (Barry, Osborne y Rose, 1996, p. 10). Son procesos de gobierno que han estudiado las prácticas y las narrativas que han fomentado nuevas subjetivaciones del homo economicus y el sujeto como empresario de sí (Han, 2014; Read, 2009). Todo lo explicitado sobre esta perspectiva de análisis ha sido manifiesto extensamente en las diferentes esferas 
sociales desde los primeros trabajos de la red de los anglofoucaultianos (Burchell, Gordon y Miller, 1991; Rose, 1999). En síntesis, los estudios de esta perspectiva de análisis, en lugar de partir de las relaciones de mercado capitalistas, se han centrado en comprender la puesta en marcha de políticas neoliberales que auto-responsabilizan al sujeto académico de sus éxitos y fracasos, bajo lógicas de mercado y competencia que son interiorizadas en los individuos.

En la actualidad, los procesos de transformación regidos por las políticas económicas del neoliberalismo estructural, así como por las racionalidades neoliberales, han sido hegemonizados en la mayor parte de las geografías. Ahora, comienzan a aparecer nuevas subjetividades regidas por prácticas de mayor libertad. Para demostrar estas dinámicas de cambio, que se extienden de forma global, en este trabajo se presentan resultados de una investigación en curso que tiene como fin comprender cómo se está modificando las subjetividades académicas en la Educación Superior a raíz de los cambios políticos recientes sobre la actividad investigadora en el contexto español y la consolidación de la industria de las publicaciones. El estudio de lo que sucede en un territorio específico, como es el caso de la universidad española, es una forma de entender cómo la neoliberalización contemporánea se desarrolla en un espacio geográfico concreto. Ejemplificar las prácticas neoliberales resituadas y readaptadas, tanto en cambios estructurales como en las subjetividades a través del análisis de una geografía política específica (Saura, 2016; Springer, 2012), es un medio para atender cómo las prácticas globales se recontextualizan en diferentes espacios.

\section{Industria de las publicaciones académicas y la sobreproducción cuantificadora de la calidad académica}

Una estandarización orientada a los resultados ha alcanzado un estatus hegemónico internacional con motivo (o al tiempo) de la implementación del llamado Proceso de Bolonia. Unos estándares de desempeño con resultados visibles, a través de las Instituciones de Educación Superior o del poder invisible de la hegemonía, mediante un currículo modular basado en resultados, obtuvieron un poder hegemónico a través de la infraestructura de la reforma de Bolonia. Como analiza y describe Brøgger (2019a), "la estandarización y la medición son partes integrales de los nuevos procesos de la política educativa europea. Son los medios por los cuales estos procesos se hacen gobernables" (p. 75). Una vez introyectados, al tiempo que, asumidos por los sujetos académicos, se convierten en una forma de gobernanza, para la que no se precisa ordenamiento legal (gobernanza hard) ni un centro legal de autoridad. Esta es la onto-epistemología, que llama Brøgger (2019a), de los nuevos procesos de gobierno: una gobernanza suave (soft), por la que el sujeto los asume como propios.

El diseño del currículo modular basado en resultados quiso instituir una moneda educativa internacional (créditos ECTS) que pudiera intercambiarse en el mercado europeo de cualificaciones. El llamado Proceso de Bolonia ha situado en primer plano los resultados y su visibilidad mediante la evaluación. El poder hegemónico del Proceso de Bolonia, incluida la forma en que los estándares se distinguen y se llevan a cabo, parece invisible porque depende de la aceptación de los contextos. Los países y universidades "ofrecen datos, intercambian formatos y se convierten en estandarizadores y, por lo tanto, en 
contribuyentes activos al poder hegemónico de la reforma y al carácter internacional de los nuevos estándares curriculares” (Brøgger, 2019b, p. 176).

En España, a medida que se implementa el proceso de Bolonia, se establece (2002) la Agencia Nacional de Evaluación de la Calidad y Acreditación (ANECA), primero como fundación del sector público y, posteriormente (2014), como Organismo Autónomo, adscrito al Ministerio, que ha contribuido decididamente a configurar, tanto para las titulaciones (acompañada de las respectivas Agencias Autonómicas), mediante el reconocimiento, lo que tiene valor en la carrera universitaria y en las vidas profesionales de los investigadores. Se impone, progresivamente, toda una burocracia: Acredita, Verifica, Modifica, competencias, sistemas de evaluación, guías para la elaboración de programas, etc. Oficialmente, su objetivo es contribuir a la mejora de la calidad del sistema de Educación Superior mediante la evaluación, certificación y acreditación de enseñanzas, profesorado e instituciones. Vamos a dejar de lado la acreditación de enseñanza e instituciones, con su exigencia de uniformidad en los formatos, para centrarnos en el profesorado.

Se ha ido imponiendo poco a poco que, la carrera académica, progresa mediante sucesivas acreditaciones, en las que se cuantifica la vida profesional, expedidas tras la evaluación por esta Agencia, aceptado e introyectado como normal. Como concluye Brøgger (2019a) su libro,

$$
\begin{aligned}
& \text { qué y cómo se mide algo es decisivo en términos de qué realidades pueden producirse } \\
& \text { a través de una reforma. La forma en que uno elige medir la progresión y la } \\
& \text { implementación contribuye a constituir el mismo ADN de una reforma. (p. 181) }
\end{aligned}
$$

La normativa no se queda en prácticas discursivas; por el contrario, su fuerza está en adentrarse como una ontología de la vida académica cotidiana, contribuyendo a configurar el nuevo sujeto académico.

El programa PEP evalúa los CV para el acceso a las figuras de profesor universitario contratado, Profesor Ayudante Doctor (PAD) y Profesor Contratado Doctor (PCD), este segundo con carácter indefinido. Por su parte, para el acceso al cuerpo de funcionarios docentes (Profesor Titular y Catedrático de Universidad) se ha establecido el programa ACADEMIA. En una primera etapa (Sistema anterior de acreditación del RD 1312/20071) se valoraba, con carácter sumativo, todas las dimensiones del currículum, lo que fue criticado por primar la cantidad sobre la calidad. Ahora con el RD 415/2015² la valoración de méritos se establece en 5 apartados: Investigación, Docencia, Transferencia, Experiencia profesional y experiencia en Gestión (se añade formación académica en el caso de Profesor Titular), dando prioridad a las dos primeras, de tal modo que, si obtiene una B en ésas dos categorías, las demás no precisan ser considerarlas. Particularmente, por su carácter excluyente (obligatorio), es la exigencia de haber publicado en revistas indexadas en JCR para obtener la acreditación en Catedrático/a (cuatro publicaciones en lo que se denomina primer cuartil) y, para poder compensar, al menos 3 JCR-Q 1. De este modo, la evaluación de la investigación, diseñada en su día para evaluar individualmente cada caso en un contex to específico, se cede a rutinarios criterios baremados. Los nuevos aspirantes

${ }^{1}$ Real Decreto 1312/2007, de 5 de octubre, por el que se establece la acreditación nacional para el acceso a los cuerpos docentes universitarios.

${ }^{2}$ Real Decreto 415/2015, de 29 de mayo, por el que se modifica el Real Decreto 1312/2007, de 5 de octubre, por el que se establece la acreditación nacional para el acceso a los cuerpos docentes universitarios. 
a docentes e investigadores universitarios se ven obligados a entrar en la carrera loca de publicar artículos en JCR o SJR, si quieren tener futuro. La exclusión de académicos con una larga trayectoria profesional, que no la han forjado bajo estas exigencias (impuestas, como tantas veces, retroactivamente) ha provocado, dos años después, la reformulación de dichos criterios para dar entrada a otras dimensiones (p. ej., monografías). Por su parte, igualmente, la CENAI, integrada en la ANECA, que reconoce los sexenios de investigación, su valoración (y concesión) se hace igualmente en función del factor de impacto de las publicaciones presentadas, sin entrar en valorar otras dimensiones relevantes de la vida académica.

La calidad medida por indicadores como el factor del impacto (eigenfactor) otorgado por las dos grandes organizaciones: Journal of Citation Reports (JCR) y Scimago Journal Rank (SJR), un índice de impacto este último elaborado a partir de Scopus. El duopolio de Clarivate y Elsevier, multinacionales que controlan el negocio de los artículos científicos, determina los criterios para su valoración. Resulta por lo menos discutible que la ANECA (en sus programas Academia o Cenai) haya abrazado, sin cuestionar, las métricas de Clarivate (antes Thompson \& Reuter) o SJR, priorizando la primera. En su lugar, se podría haber optado, al menos de modo complementario, por otras métricas responsables, que complementen o equilibren el valor académico o científico de una contribución. La facilidad de su aplicación, así como la no posibilidad de cuestionamiento, dado que basta contar el index y el número, no obsta para mostrar sus graves limitaciones. Entre otros, no se entra en la valía de lo publicado o en su impacto, dicha valoración se cede a las agencias JCR o SJR, en un sistema burocrático, que se limita a cuantificar.

Ante esta situación, contra natura, una sentencia del Tribunal Supremo (12/06/2018) determina que:

Las investigaciones, las aportaciones presentadas por los interesados, no pueden dejar
de examinarse solo por el hecho de que no se publicaran en las revistas o medios
incluidos en los índices o listados identificados $[\ldots]$. Ni tampoco están excluidos por
esa sola razón de la máxima valoración permitida [...]. Dependerá de su contenido
la evaluación que merezcan.

Sin embargo, dicha sentencia no ha tenido efectos en lo establecido. Por su parte, de modo paralelo, la evaluación de la actividad investigadora por la CENAI (incluida ahora en la ANECA), vinculada a incentivos económicos mediante los conocidos tramos o sexenios de investigación, progresivamente ha supuesto un reconocimiento académico a profesores e investigadores, condicionando además de forma directa la progresión en su carrera académica o, paralelamente, a disminuir su carga docente. Ha continuado, además, la minusvaloración, en la práctica, de dejar sin valor el ensayo o libros, por la multiplicación de papers, en formato de artículo, lo que hace que los mejores escritores (George Steiner u Ortega y Gasset,) no superasen los números, favoreciendo a los contables de la estadística y no a los estudiosos humanistas que escriben excelentes monografías. Por su parte, siguiendo el principio de que el contenido no importa, sino el medio en que se publica, se necesitaban indicadores de las monografías o libros. En el ámbito español se han publicado unos indicadores, producto de unos Proyectos de Investigación, llamados SPI (Scholarly Publishers Indicators) en función de un conjunto de indicadores, entre los cuales ocupa el primer lugar el prestigio editorial. Reconociendo la relevancia de este trabajo, en un campo

s Tribunal Supremo (Sala de lo Contencioso Administrativo, Sección 4a). Sentencia núm. 986/2018 de 12 de junio. 
dejado al azar, para clarificar este amplio territorio, también es preciso resaltar sus limitaciones. Obviamente no se entra en la relevancia de cada libro ni en su contribución al progreso del conocimiento, solo se valora el editor. Como hemos señalado antes, además, se viene abajo cuando se ha ido convirtiendo en una práctica generalizada de pagar para publicar.

Ante este panorama, otras métricas alternativas son posibles y necesarias. Como manifestación de esta necesidad de mejorar la forma de evaluación de la calidad científica de las revistas, por una parte, cada país está desarrollando sus propios modelos (así, por ejemplo, QUALIS en Brasil, o sello de calidad FECYT en España) o regionales (como métricas DIALNET para revistas españolas de Ciencias Sociales y Humanidades). A nivel general, la Declaración de San Francisco sobre Evaluación de la Investigación (DORA) es hasta ahora el impulso mayor a este otro tipo de métricas alternativas o más amplias. De hecho, algunas plataformas como REDALYC exigen para incluir una revista que esté adherida a DORA. La Declaración de Berlín sobre Acceso Abierto al Conocimiento en Ciencias y Humanidades o el Manifiesto de Leiden (Hicks et al., 2015) son algunas de las manifestaciones y llamadas sobre otras métricas son posibles, con la exigencia de estándares de rigor y práctica científica solvente, con mecanismos de control del contenido publicado transparente y riguroso (revisión por pares) y que no impliquen ningún cargo ni tasa para poder publicar. Este Manifiesto de Leiden, promovido por prestigiosos científicos, y publicado en Nature, finalizaba Como "no se debe permitir que la información cuantitativa se convierta en un objetivo en sí misma. Las mejores decisiones se toman combinando estadísticas robustas sensibles a los objetivos y la naturaleza de la investigación evaluada" (Hicks et al., 2015, p. 431).

Si en la modernidad, como recuerda Habermas (1987), la investigación en el modelo ideal diseñado por Humboldt para la Universidad de Berlín, era un ámbito puro e incontaminado; ahora esto se ha visto gravemente cuestionado. La corrupción mercantilista y capitalista está invadiendo el trabajo académico y, lo peor, que se está viendo como natural o inevitable. Los negocios en torno a cómo hacer visible el currículum académico de modo rápido proliferan, lo que pone de manifiesto el deterioro de la ciencia por la necesidad de evidenciar una sobreproducción (varios investigadores se ponen de acuerdo para poner sus nombres mutuamente y de este modo multiplicar uno por cuatro). De este modo, la necesidad de publicar (pronto y rápido) ha generado, de modo cada vez más extendido, el pago y negocios financieros con las publicaciones en revistas depredadoras (Beall, 2018).

Jeffrey Beall (2017) habla del colapso de las publicaciones académicas, como manifiestan tanto la proliferación creciente de revistas depredadoras como el pagar por publicar, cada vez más generalizado en el mundo editorial español e internacional. El asunto no es tanto el pago, sino que el criterio primero sea el pago (author-pays), no la calidad y revisión académica previa por pares de su relevancia científica. Las revistas depredadoras se convierten en repositorios donde los autores pagan para que sus documentos se conviertan en documentos .pdf, colocados bajo el nombre de una revisa en Internet. Como fuente potencial de ingresos, la industria editorial académica se ha entregado los editores depredadores. Como dice un buen conocedor:

La industria editorial académica no tiene un sistema de credencialización, ningún control de calidad, y muchas de las empresas de apoyo editorial, como Crossref (el proveedor de identificadores de objetos digitales, DOI), acogen con satisfacción las revistas depredadoras como fuentes de ingresos adicionales. (Beall, 2017, p. 277) 
En fin, el acuerdo tácito hasta ahora existente (mantener altos niveles de integridad y exigencia académica en las publicaciones) se ha abandonado, con una complicidad y consentimiento mutuo (editores y autores). Si este es el juego existente, se viene a decir, juguémoslo, si no queremos quedar fuera.

Si necesita publicarlo pronto (preprint, online first), con DOI incluido, debe pagar. Por eso están creciendo exponencialmente. Justificado dicho pago como parte del coste (Article publishing charge), sin embargo, justamente por ser en abierto, se ha convertido en todo un (Publishing fees). Negocio ya no solo practicado por editores chinos que cobran en francos suizos (por ejemplo, MPDI), sino en el que han entrado las editoriales más prestigiosas. Así, como ejemplo conocido personalmente, la revista Studies in Higher Education (JCRQ1), editada por Taylor \& Francis, ha sacado una versión en Open Access por la que cobra (según contenido y figuras) varios miles de euros.

Este fenómeno que describimos se inscribe más ampliamente, como nos hemos referido antes, en lo que Slaughter y Leslie (2001) llamaron capitalismo académico, por el que hay un desplazamiento de un régimen de conocimiento público a otro de carácter privado. Brunner y su equipo (2019) acaban de hacer una revisión del concepto y su extensión en América Latina. Por más que nos lamentemos la Universidad y la Investigación alejada en su torre de Marfil ha dejado de existir. Habermas (1987), desde la mejor tradición moderna y alemana, reivindica a una Universidad, como servicio público, para la formación de ciudadanos críticos, competentes profesionales y con una deontología profesional, ahora periclitada. Frente a los efectos colonizadores del sistema (estructuras económicas y de poder), señalaba: "El sistema científico diferenciado no se debe fusionar justamente, sólo con la economía, la técnica y la administración, sino que debe quedar enraizado más allá del vínculo tradicional de sus funciones en el mundo de vida” (p. 10). Sin alimentar ninguna nostalgia académica de tiempos pasados, antes que sea tarde, ha llegado el momento de decir que no.

En una conferencia de Antonio Nóvoa (ECER, Porto, 2014), ahora publicada (Nóvoa, 2015), sobre el futuro que aguarda a la investigación educativa, se hacía eco de esta situación. Tenemos diferentes casos que expresan el malestar más profundo, que afecta la vida académica y universitaria. Signos que se han multiplicado a un ritmo alarmante en los últimos años, síntomas de corrosión. Este tipo de prácticas actuales, "están destruyendo nuestra vida académica y nuestra libertad intelectual. Estas ideologías están empobreciendo el trabajo científico, en particular en el campo de la investigación en educación” (p. 269).

\section{Método de trabajo}

¿Cómo se configura el sujeto académico neoliberal? ¿Cómo en la academia neoliberal los sujetos son auto-seducidos mediante estrategias de libertad en lugar de ser dirigidos mediante elementos de control y conducción de las conductas? ¿Cómo los académicos se relacionan con los otros y consigo mismos a través de estos nuevos procesos de gubernamentalidad neoliberal? Estos interrogantes iniciales han sido los puntos de partida para investigar e intentar comprender las dinámicas de cambio y los procesos de configuración de los académicos en la Universidad actual. Son interrogantes cotidianos en cualquier encuentro entre académicos que remiten a la comprensión de los cambios de 
gubernamentalidad dentro de las universidades y a la configuración de las nuevas subjetividades académicas.

Los interrogantes planteados, destinados a la comprensión hermenéutica de los nuevos procesos de subjetivación, conducen a plantear la investigación desde un enfoque cualitativo, en tanto que se pretende profundizar en los discursos de los actores, siendo las estrategias cualitativas las formas más eficaces de relejar las singularidades de los procesos de construcción subjetiva. La presente investigación se sustenta bajo un enfoque cualitativo que se desarrolla bajo un marco de investigación interpretativo y un método de análisis inductivo que está en proceso constante de cambios según los avances de la investigación (Hernández, 2014). Bajo este marco cualitativo-interpretativo, la investigación se apoya en la interpretación de discursos extraídos mediante la técnica de entrevistas semiestructuradas en profundidad, como proceso dialógico de investigación (Kvale, 2011), realizadas a 24 académicos/as de universidades españolas.

Durante el proceso de análisis las narrativas comenzaron a reiterar las informaciones que se había recogido previamente, alcanzando así una saturación teórica de los discursos, lo que posibilitó paralizar el proceso de recolección de datos en esta fase. La selección de las personas participantes se ha realizado mediante el empleo de la técnica snowball sampling (Noy, 2008). Esta técnica parte de un sujeto, también entendido en el marco cualitativo como caso, que reúne características interesantes para el objeto de estudio. Para esta investigación se consideraron dos dimensiones que condujeron posteriormente al total de participantes de la investigación: experiencia profesional y número de publicaciones. Se ha entrevistado a dos hombres y dos mujeres por cada una de las siguientes categorías en el campo de la educación: Estudiante de Doctorado (ED); Profesor Asociado (PA); Profesor Ayudante Doctor (PAD); Profesor Contratado Doctor (PCD); Profesor Titular (PT); Catedrático Universitario (CU). Junto a ello, en la investigación se desarrolla un análisis crítico del discurso (Van Dijk, 2008) de políticas, documentos, normativas y debates de diversos actores políticos en la política universitaria española que se ha dejado de lado en este texto.

El proceso de análisis, iniciado desde la primera entrevista realizada, conformó categorías de partida, iniciales o vivas, extraídas de una codificación de modalidad inductiva, por lo que se aplicaron nomenclaturas fieles al discurso de los participantes. Siguiendo el proceso de bricolaje del investigador, característico de la investigación cualitativa, estas categorías iniciales se fueron integrando en nodos narrativos más amplios. De este modo, aparecieron ocho categorías principales en los extractos discursivos de los informantes: cuantificación, perfomatividad, somatización, sujeto errático, ambivalencia, auto-seducción, digitalizaciones, bibliometrificación. Estas categorías son los puntos nodales que articulan el apartado de los resultados y conclusiones.

En este texto se plasma algunas de las voces de los participantes, utilizando una combinación de las perspectivas emic (narrativa de los participantes) y etic (interpretación que el investigador hace de dichas narrativas) (Sugrue, 2015). Para ello, construimos la interpretación de los discursos a partir de dos aspectos fundamentales. Por un lado, nos centramos en la interpretación de la influencia de la cuantificación de la producción en la modificación de la conducta académica y la somatización del neoliberalismo en los académicos. Y, por otro, exploramos bajo nuestra interpretación las formas en los que los académicos se constituyen en sujetos bibliometrificados y digitalizados. 


\section{La cuantificación de la producción académica y la somatización del neoliberalismo}

A través de las dinámicas de publicar o perecer, popularizada por Harzing, se han analizado las estrategias que durante las dos últimas décadas las universidades han desarrollado para que los académicos aumenten las publicaciones para poder competir en la arena global del conocimiento (Harzing, 2010). Estos cambios se han consolidado mediante una gobernanza interrelacionada entre la industria de las publicaciones, el afán por competir entre las universidades y las mejoras en las condiciones laborales de los académicos según las publicaciones. Todo ello, que ya forma parte del día a día de cualquier institución universitaria, ha producido la consolidación de la época dorada de las publicaciones académicas.

Los efectos del publicar o perecer, así como de lo que se ha tratado en el apartado anterior sobre la sobreproducción de las publicaciones y el régimen de la cuantificación tienen su precio en el sujeto. La noción de performatividad, como tecnología política esencial de la gubernamentalidad neoliberal en educación (Ball, 2012; Luengo y Saura, 2013), ha sido un apoyo conceptual para estudiar algunas de las consecuencias de la cuantificación desde los primeros trabajos que ha abordado las racionalidades neoliberales en Educación Superior (por ejemplo, Barnett, 1996; Cowen, 1996). La performatividad es la lógica que hace al sujeto eficiente, eficaz y determinista que actúa siempre bajo la búsqueda incansable de la producción cuantificadora. Es una tecnología política que se adentra en el sujeto para dirigir sus acciones de modo finalista bajo el rendimiento constante de la sobreproducción.

No obstante, en la actualidad, una vez se han consolidado las lógicas de actuación de la tecnología política de la performatividad en el ámbito académico, ya no es necesario ejercer un control externo de la evaluación. En estos momentos, por lo que Mirowski (2013) ha capturado con finura como everyday neoliberalism (neoliberalismo cotidiano o neoliberalismo diario), el sujeto tiene interiorizadas nuevas prácticas de auto-control de sí. Son prácticas mundanas en las que los individuos actúan bajo lógicas del individualismo y la competencia contra otros/as y contra sí, desarrolladas por estrategias de mayor libertad. La voz de un joven que recientemente ha logrado una plaza de Profesor Ayudante Doctor, dice de forma muy simple, mucho sobre ello:

\section{Nosotros trabajamos cuatro. Cada uno hace un artículo y ponemos el nombre de los cuatro en cada paper. (PAD1)}

Son formas de perversión del conocimiento, imperantes en la institución universitaria, muy cotidianas actualmente. La finalidad es la producción cuantificada del conocimiento. El saber no es nada y la cantidad lo es todo. Como expresa un estudiante de doctorado de segundo año, ya no importa el conocimiento logrado ni la dedicación plena a la tesis doctoral; toda meta reside en el producto:

\footnotetext{
Yo trabajo sin parar para entrar con una plaza de ayudante [PAD], una vez estás dentro, ya leeré y haré las cosas distintas. Mientras, tengo que producir y producir. Tú no sabes cómo está el departamento, los que entran... (ED2)
}

La experiencia, la calidad de las publicaciones y los hallazgos en el doctorado ya no son metas que identifiquen al buen académico. La cuantificación de la productividad es ahora un valor esencial que dignifica al sujeto. La solidez y la rigurosidad del trabajo, que reclama una o pocas líneas de investigación académicas, no es propio del sujeto académico contemporáneo. El deseo de la cuantificación, el afán por poseer más y más artículos, 
aunque no sean de calidad; es decir, la apuesta incesante por la cantidad en lugar de la calidad, es muy propio de estos tiempos. Una de las consecuencias es que la configuración de los sujetos académicos se dirime entre la sobreproducción de la escritura y la escasez en la lectura (Altbach y de Wit, 2019).

No he leído ni un libro completo en el doctorado. Leo solamente artículos, y muchos solamente abstracts. Muchas veces descargas los textos sabiendo lo que buscas. (ED2)

Ya leeré cuando tenga la plaza. Ahora lo importante es producir. [...] Si algún compañero o compañera me propone un trabajo publicable, yo digo que sí, más de una vez no sé nada de la temática. Siempre puedo aportar sobre la metodología o hacer una revisión sistemática de la literatura. (ED4)

La sobreproducción de las publicaciones y la mirada siempre finalista de la cuantificación tiene su precio también en lo que importa: los otros cercanos; y sobre sí. Un extracto de la conversación que Rosallind Gill (2010) mantuvo con una colega es un buen ejemplo para expresar algunas de estas consecuencias muy visibles en el día a día de nuestras universidades:

A mí me ocurre lo mismo. ¿Leer? ¿Qué es eso? ¿Pensar? ¡Ninguna posibilidad! $\Upsilon$ te sientes fatal, ¿̇no te parece? En mi caso, además, siento como si constantemente les robara el tiempo a los niños [...] En ocasiones siento como si les estuviera abandonando. (p. 1)

Algo similar relata un profesor titular cuando tiene una mirada retrospectiva sobre su experiencia vivida respecto a la sobreproducción académica para el logro de la acreditación:

La Universidad te engaña. Me pilló el cambio de las acreditaciones. Me tuve que dedicar a hacer artículos, en vez de libros, los mejores años de mi vida. No pude ver cómo mis hijos crecían por la acreditación y la titularidad. [...] No hay nada de lo que más me arrepienta. Te digo esto porque te aprecio y eres joven. Tú eres un intelectual, pero tienes que vivir. Luego pasarán los años y cuando mires atrás te arrepentirás, como me pasó a mí... (PT1)

Además de la sobreproducción y las lógicas del individualismo, que provoca dejar de lado lo que importan, los efectos van estrechamente ligados a nuevos procesos de somatización del neoliberalismo en el propio yo. El estrés, la ansiedad y el insomnio, aunque no se suelen expresar con regularidad en los encuentros académicos, son efectos directos muy comunes de estas lógicas de la universidad neoliberal.

He experimentado mareos, he tenido dolor en el pecho, sensaciones de angustia, de...pfff. Me ha cambiado la actitud [...] He experimentado vértigos. Cuando estoy con un proceso de vértigos que no puedo levantarme por los mareos que sufro, tengo que ir constantemente al médico. Cuando esos mareos ya no responden a proceso de vértigos, sino que hay palpitaciones, taquicardias, sensaciones de desmayo... (ED3)

$\mathrm{Al}$ acudir a diversos profesionales médicos, este participante nos relata que los análisis clínicos han determinado que padece problemas graves de salud derivados de episodios de ansiedad. Son ejemplos visibles de los efectos negativos en la salud de la somatización del neoliberalismo por las lógicas de la producción cuantificadora académica. La somatización del neoliberalismo en la academia es una epidemia. No son efectos únicamente de la universidad española. Como han demostrado recientemente Guthrie y colaboradores (2018) el deterioro de la salud mental entre los académicos en Reino Unido ya representa índices similares a los que posee el personal de profesiones consideradas de riesgo. $\mathrm{El} 40 \%$ de los jóvenes encuestados en el estudio de Reino Unido presentaron síntomas de depresión, problemas relacionados con las emociones y altos niveles de estrés. Las 
generaciones jóvenes son las más afectadas debido a la vulnerabilidad, la precariedad y la competición que existe para lograr un contrato de estabilidad laboral. Es algo muy común padecer estos episodios de estrés y ansiedad debido a las lógicas de la sobreproducción académica que originan la presión de la competición entre candidatos para poder acceder a un puesto de trabajo que se rige únicamente por méritos basados en una meritocracia cuantitativa.

La ansiedad, la despersonalización, el escribir por escribir; por la presión. $\Upsilon$ sobre todo de escribir no disfrutando de la escritura, y por consiguiente no disfrutando de la lectura, sino sufriendo con esos procesos. (PAD3)

La vida psíquica del neoliberalismo es como Scharff (2017) define los procesos de somatización derivados de las dinámicas cuantificadoras de la sobreproducción académica.

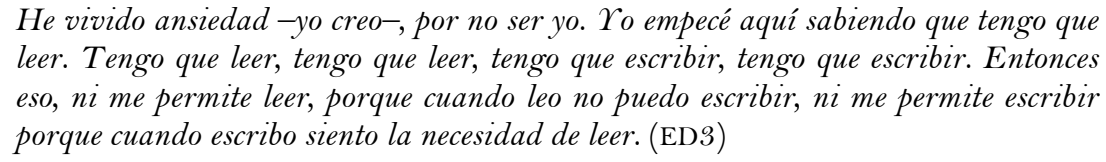

Estos dos últimos extractos representan aspectos de un ser errático y ambivalente que vive entre dos polos. En uno, está su ética: lo que realmente quiere hacer (leer, adquirir conocimiento). En otro lado -predominando-, están las acciones constantes de sobreproducción cuantificada de las publicaciones. Son prácticas neoliberales contemporáneas que se sustentan en la configuración de un académico errático y ambivalente que se mueve continuamente de sus pretensiones, para reproducir las exigencias del sistema que no son las que desea hacer. Esta ambivalencia ejemplifica resultados similares a lo que ha sido descrito como la creación de nuevas subjetividades académicas de la universidad esquizofrénica o de trastornos de personalidad múltiple (Shore, 2010, p. 20) que comienza a producir académicos neuróticos (Loveday, 2018). Son procesos de somatización del neoliberalismo que se introducen en la psique del sujeto constituyendo un sujeto académico ambivalente. Queda muy clarificado en esta voz:

\begin{abstract}
Ahora estoy sufriendo con estos procesos. Ahora estoy sufriendo cuando leo porque si leo considero que no produzco, si no produzco no me acredito, si no me acredito no entro a la Universidad. Si escribo no estoy leyendo, por tanto, lo que escribo no es valioso, por tanto, eso no tiene un impacto sobre lo que yo quiero estudiar. To pienso en dejarlo muchas veces. Unas veces subes y otras bajas. La semana pasada, cuando me rechazaron el artículo, lo único que quise es dejar la Uni... (ED3)
\end{abstract}

\title{
5. Sujetos académicos bibliometrificados y digitalizados
}

Recientemente, comienza a aparecer un amplio conjunto de investigaciones muy llamativas que evidencian nuevas subjetividades en la academia neoliberal por el uso cotidiano de los índices de impactos bibliométricos y las plataformas digitales que miden la calidad de los individuos mediante cuantificaciones digitalizadas (Feldman y Sandoval, 2018; Lupton, 2016; Lupton, Mewburn y Thomson, 2018). Son investigaciones que nacen para prestar atención a la "creciente gama de tecnologías, particularmente aquellas relacionadas con la contabilidad y la gestión de los datos, que se utilizan actualmente en la gobernanza de la educación superior" (Lim, 2019, p. 2). Estos estudios conforman un nuevo campo de análisis para comprender los cambios que las plataformas digitales y los dispositivos tecnológicos están ejerciendo como nuevos procesos de control que monitorean la valía y los comportamientos de los individuos en todos los aspectos de la vida. Mediante las nociones de self-tracking y quantified self, Deborah Lupton (2016) ha 
explorado con intensidad los modos bajo los que los individuos auto-cuantifican sus conductas mediante procesos de datificación y digitalización en el mundo digital, que conforman al sujeto académico digitalizado (Lupton, Mewburn y Thomson, 2018). En las esferas del deporte, la salud, la alimentación y lo social, por poner algunos ejemplos, es algo muy común que el sujeto auto-cuantifique y haga visibles los datos de las actividades cotidianas mediante plataformas digitales y el poder de las métricas (Beer, 2016; Feldman y Sandoval, 2018).

Mucho tiene que ver con todos estos cambios en el mundo académico la aceptación que ha tenido la creación del índice h que impulsó Hirsch en el año 2005. Se trata de un factor de impacto basado en la lógica simplista de que "un científico tiene índice h si h de sus $N p$ papers recibe al menos h citas cada uno, y los otros $(N p h)$ papers tienen como máximo $\mathrm{h}$ citas cada uno" (Hirsch, 2005, p. 1). El índice h se consolida como una medida de la valía del sujeto. Incluso en los procesos de evaluación de la calidad académica y en las memorias del CV, que hay que rellenar en las convocatorias de proyectos de investigación nacionales en España, las citas y el factor de impacto de cada individuo se utiliza como indicador cuantitativo de la calidad. El uso activo que hace el sujeto académico de esta medida de impacto se ejerce a través de prácticas de cuantificación y visibilización de la producción que son guiadas libremente por los académicos. Ahora cada sujeto cuida su Google Scholar, selecciona las publicaciones que quiere hacer visibles para exponer la imagen que quiere transmitir de sí al exterior. Tiene que ver con lo Hall (2016) ha llamado el selfbranding o marca personal de la Uberficación académica. Cuando el académico siente vergüenza por lo que ha escrito previamente y quiere renovar su marca personal, elimina las publicaciones que ya no le representan para rehacerse en un nuevo sujeto visibilizado al exterior:

Me he cerrado la cuenta de Google Scholar porque no me sentía cómodo con esas publicaciones, no me sentía cómodo con los tópicos que tenía asignados a mi perfil, porque la investigación te lleva constantemente a cosas diferentes a la que tú planteabas. Mi decisión fue: fuera. [...]. Ahora pondré las áreas que ya tengo consolidadas. Voy a cuidarlo. Mi finalidad es meter ahi las que me representan, las que estoy investigando ahora. No me representan las publicaciones que he hecho para tener méritos. (ED4)

Junto a Google Scholar, otras redes sociales y plataformas digitales tales como Orcid, Twitter, Academia.edu o Researchgate son algunos de los canales más comunes mediante los que el sujeto académico digitalizado hace visible su rendimiento. El sujeto académico auto-cuantificado cuida su imagen en las redes sociales mediante la huella de uno/a mismo/a. Estas plataformas digitales representan en la actualidad nuevos procesos de subjetivación neoliberal, donde el propio sujeto produce una marca propia como medio para auto-monitorearse y auto-promocionarse hacia el exterior. Es un mecanismo de control que está muy alejado de la imposición; es un mecanismo de auto-control regido por la seducción, como nos relatan sobre Researchgate:

Cuando alguien consulta mis textos me siento bien. Si consideran que es bueno o malo, lo decidirá la gente, pero me siento contento cuando lo leen. Me satisface que personas que yo respeto y admiro en el ámbito académico están consultando mi producción [...] Sí que me alegra cuando lo leen Michael Apple o Thomas Popkewitz, o cualquier de los profesores que he tenido en la universidad, me hace enteramente feliz. (ED3)

La voz de este participante tiene que ver con el ego-ideal que hablan Moore y Robinson (2016), donde la "subjetivación toma una forma particular en el neoliberalismo, en el cual los sujetos se autodefinen en términos de su estatus para la mirada cuantificada externa" 
(p. 3). Estas prácticas de visibilización que hace el sujeto digitalizado contemporáneo emiten nuevos procesos control, más allá de lo que Foucault sintetizó como tecnologías del yo. Ahora, los procesos de gubernamentalidad neoliberal son más sutiles que antes, ya que los sujetos son auto-monitoreados y auto-controlados desde la libertad engañosa de la auto-seducción de la neoliberalización contemporánea en la academia.

\begin{abstract}
To abro casi las mismas veces Academia.edu y Researchgate que el correo electrónico. Mis cuentas las cuido, tengo muchas visitas, tengo mis mejores textos. Uso las visualizaciones que tengo en el $\mathrm{CV}$, como impacto de mi trabajo. Ahora estoy entre el top one de los más visitados de todo el mundo. Mi imagen, mis followers y a los que sigo es muy importante. (PAD3)
\end{abstract}

El resultado es la producción de un "yo" exitoso que es "flexible, modular y plástico", como características esenciales del germen del "neoliberalismo cotidiano" (Mirowski, 2013, p. 108). Una línea de subordinación que va del sistema económico a la imagen ego-ideal o subjetiva; donde se seduce al individuo para que "use su mente para subordinar su cuerpo al ideal del ego y, por lo tanto, al sistema económico" (Moore y Robinson, 2016, p. 2). Un sujeto bibliometrificado que se cuida de sí teniendo como meta el logro de configurarse en un sujeto mensurable, medible y cuantificable a través de la digitalización. Como un proceso de gobierno desde la distancia, se produce un "sujeto bibliometrificado" (Lim, 2019 , p. 2) que ya no es disciplinado ni conducido desde el exterior, sino que sigue permanentemente auto-seducido desde su propio yo. El resultado es un sujeto visible y visibilizado, pero vacío.

\title{
6. Conclusiones
}

Una vez hegemonizados los procesos estructurales de la globalización neoliberal en la Educación Superior a través de políticas de liberalización económica, competencia entre países y rankings (De Angelis y Harvie, 2009; Slaughter y Leslie, 2001), así como las políticas de racionalidades neoliberales académicas que individualizan al sujeto de sus éxitos y fracasos a través de evaluaciones (Cannizzo, 2015) comienzan a aparecer nuevos modos de gobernanza más sutiles que modifican las subjetividades académicas. En esta investigación inicial sobre la temática, con las limitaciones que contiene esta muestra exploratoria y contextualizada, hemos intentado acercar la interpretación sobre unas narrativas que demuestran cómo las nuevas tecnologías políticas de la bibliometría, las digitalizaciones y las cuantificaciones de la producción académica (Lupton, Mewburn y Thomson, 2018) están logrando constituir nuevas subjetividades neoliberales, que ya no necesitan estar sometidas a un control externo ni tampoco a una conducción de las conductas desde el exterior. Estas dinámicas de cambio logran efectuar procesos de la neoliberalización de los académicos que comienzan a operar mediante estrategias fundadas en la auto-seducción generada por el propio sujeto, siempre bajo nuevas prácticas de un modus operandi regido por una mayor libertad. Las prácticas neoliberales diarias constituyen un sujeto que se auto-controla a sí mismo mediante una sobreproducción de la cantidad de publicaciones académicas y a través de un cuidado de sí de su imagen visible exteriorizada. El ejercicio de estas prácticas se hace ahora de modos más libres y solitario que antes, y tiene sus consecuencias en el conocimiento académico y en el propio yo.

La sobreproducción de artículos, el factor de impacto, el índice h, las evaluaciones cuantificadoras, las acreditaciones de agencias de calidad, la obsesión por aumentar el cV, la visibilidad externa en Google Scholar, Researchgate y Academia.edu, etc., se convierten en el devenir del sujeto ocasionando una serie efectos incontrolados de somatización del 
neoliberalismo, tales como: el estrés, el insomnio, la ansiedad, el sinsentido académico, la ambivalencia profesional, el individualismo y la competición desmedida. Todo ello compone un conjunto de prácticas y efectos que configuran al sujeto académico neoliberal de la universidad contemporánea. Los académicos centran sus acciones en producir, producir y producir, y su éxito profesional queda determinado por la obtención o no de proyectos, la publicación o no de artículos y la consecución o no de acreditaciones, sin importar su trayectoria ni experiencia previa. Se produce así la crisis de una identidad que queda fragmentada entre el querer ser y el deber hacer. La identidad académica, ya no procede de un orden establecido, sino un devenir en construcción de "un proyecto a realizar" (Bolívar, 2006, p. 11) que ofrece oportunidades a la comunidad investigadora para profundizar en las prácticas mundanas de nuestro presente en la academia contemporánea.

\section{Referencias}

Altbach, P. G. y de Wit, H. (2019). Too much academic research is being published. International Higher Education, 96, 2-3. https://doi.org/10.6017/ihe.2019.96.10767

Badiou, A. (2008). Number and numbers. Cambridge, MA: Polity Press.

Ball, S. J. (2012). Performativity, commodification and commitment: An I-spy guide to the neoliberal university. British Journal of Educational Studies 60(1), 17-28. https://doi.org/10.1080/00071005.2011.650940

Barnett, R. (1996). The evaluation of the higher education system in the United Kingdom. En R. Cowen (Ed.), The world yearbook of education: The evaluation of higher education systems: Londres: Kegan Paul.

Barry, A., Osborne, T. y Rose, N. (1996). Foucault and political reason: Liberalism, neoliberalism and rationalities of government. Chicago, IL: University of Chicago Press.

Beall, J. (2017). What I learned from predatory publishers. Biochemia Medica, 27(2), 273-278. https://doi.org/10.11613/BM.2017.029

Beall, J. (2018). Scientific soundness and the problem of predatory journals. En A. Kaufman y J. Kaufman (Eds.), Pseudoscience: The conspiracy against science (pp. 283-299). Cambridge, MA: The MIT Press. https://doi.org/10.7551/mitpress/9780262037426.003.0012

Beer, D. (2016). Metric power. Londres: Palgrave Macmillan. https://doi.org/10.1057/978-1-137$55649-3$

Brøgger, K. (2019a). Governing through standards: The faceless masters of higher education. Cham: Springer. https://doi.org/10.1007/978-3-030-00886-4

Brøgger, K. (2019b). How education standards gain hegemonic power and become international: The case of higher education and the Bologna process. European Educational Research Journal, 18(2), 158-180. https://doi.org/10.1177/1474904118790303

Brunner, J. J., Labraña, J., Ganga, F. y Rodríguez-Ponce, E. (2019). Idea moderna de universidad. De la torre de marfil al capitalismo académico. Educación XX1, 22(2), 119-140. https://doi.org/10.5944/educxx 1.22480

Burchell, G., Gordon, C. y Miller, P. (1991). The Foucault effect: Studies in governmentality. With two lectures by and an interview with Michel Foucault. Hemel Hempstead: Harvester Wheatsheaf. https://doi.org/10.7208/chicago/9780226028811.001.0001

Butler, J. (2010). Mecanismos psíquicos del poder. Madrid: Cátedra. 
Cannizzo, F. (2015). Academic subjectivities: Governmentality and self-development in higher education. Foucault Studies, 20, 199-2 17. https://doi.org/10.22439/fs.voio.4937

Cheney-Lippold, J. (2017). We are data: Algorithms and the making of our digital selves. Nueva York, NY: New York University Press. https://doi.org/10.2307/j.ctt1gk0941

Cowen, R. (1996). Performativity, Post-modernity and the university. Comparative Education, 32(2), 245-258. https://doi.org/10.1080/03050069628876

De Angelis, M. y Harvie, D. (2009). Cognitive capitalism and the rat-race: How capital measures immaterial labour in British universities. Historical Materialism, 17(3), 3-30. https://doi.org/10.1163/146544609X12469428108420

Feldman, Z. y Sandoval, M. (2018). Metric power and the academic self: Neoliberalism, knowledge and resistance in the British university. TripleC, 16(1), 214-233. https://doi.org/10.31269/triplec.v16i1.899

Foucault, M. (1988). El sujeto y el poder. Revista Mexicana de Sociología, 5o(3), 3-20. https://doi.org/10.2307/3540551

Foucault, M. (1991). Gubernamentalidad. En AA.VV. Espacios de poder (pp. 9-26). Madrid: La Piqueta.

Foucault, M. (2007). Nacimiento de la biopolítica. Curso en el Collège de France (1978-1979). Buenos Aires: FCE.

Gill, R. (2010). Breaking the silence: The hidden injuries of neo-liberal academia. En R. Flood y R. Gill (Eds.), Secrecy and silence in the research process: Feminist reflections (pp. 228244). Londres: Routledge.

Guthrie, S., Lichten, C., Van Belle, J., Ball, S., Knack, A. y Hofman, J. (2017). Understanding mental health in the research environment. A rapid evidence assessment. Santa Mónica, CA: RAND Corporation. https://doi.org/10.7249/RR2022

Habermas, J. (1987). La idea de la universidad-procesos de aprendizaje. Sociológica, 5(2), art 1. Recuperado de

http://www.sociologicamexico.azc.uam.mx/index.php/Sociologica/article/view/1045/10 17.

Hall, G. (2016). The uberfication of the university. Minneapolis, MI: University of Minnesota Press. https://doi.org/10.5749/9781452958439

Han, B. (2014). Psicopolítica. Neoliberalismo y nuevas técnicas de poder. Barcelona: Herder.

Harzing, A. W. (2010). The publish or perish book. Melbourne: Tarma Software Research.

Hernández, R. M. (2014). La investigación cualitativa a través de entrevistas. Su análisis mediante la teoría fundamentada. Cuestiones Pedagógicas, 23, 187-210.

Hicks, D., Wouters, P., Waltman, L., De Rijcke, S. y Rafols, I. (2015). The Leiden manifesto for research metrics. Nature, 520, 429-431. https://doi.org/10.1038/520429a

Hirsch, J. E. (2005). An index to quantify an individual's scientific research output. Proceedings of the National Academy of Sciences, 102(46), 16569-16572. https://doi.org/10.1073/pnas.0507655102

Howie, G., (2005). Universities in the UK: Drowning by numbers. Critical Quarterly, 47, 1-10. https://doi.org/10.1111/j.0011-1562.2005.00610.x

Jankowski, N. y Provezis, S. (2014). Neoliberal ideologies, governmentality and the academy: An examination of accountability through assessment and transparency. Educational Philosophy and Theory, 46(5), 475-487. https://doi.org/10.1080/00131857.2012.721736 
Kvale, N. (2011). Las entrevistas en la investigación cualitativa. Madrid: Morata.

Lim, M. A. (2019). Governing higher education: The PURE data system and the management of the bibliometric self. Higher Education Policy, 1-16. https://doi.org/10.1057/s41307-01800130-0

Loveday, V. (2018). The neurotic academic: Anxiety, casualisation, and governance in the neoliberalising university. Journal of Cultural Economy, 11(2), 154-166. https://doi.org/10.1080/17530350.2018.1426032

Luengo, J. y Saura, G. (2013). La performatividad en la educación: La construcción del nuevo docente y el nuevo gestor performativo. REICE. Revista Iberoamericana sobre Calidad, Eficacia y Educación, 11(3), 139-153.

Lupton, D. (2016). The quantified self: A sociology of self-tracking. Cambridge: Polity Press.

Lupton, D., Mewburn, I. y Thomson, P. (2018). The digital academic: Critical perspectives on digital technologies in higher education. Milton Keynes: Routledge.

Lyotard, J. F. (1979). La condición postmoderna. Madrid: Cátedra.

Marginson, S. (2013). The impossibility of capitalist markets in higher education. Journal of Education Policy, 28(3), 353-370. https://doi.org/10.1080/02680939.2012.747109

Mirowski, P. (2013). Never let a serious crisis go to waste: How neoliberalism survived the financial meltdown. Londres: Verso.

Moore, P. y Robinson, A. (2016). The quantified self: What counts in the neoliberal workplace. New Media E Society, 18(11), 1-19. https://doi.org/10.1177/1461444815604328

Morrissey, J. (2013). Governing the academic subject: Foucault, governmentality and the performing university. Oxford Review of Education, 39(6), 797-810. https://doi.org/10.1080/03054985.2013.860891

Nóvoa, A. (2015). Em busca da liberdade nas universidades: Para que serve a pesquisa em educação? Educação e Pesquisa, 41(1), 263-272. https://doi.org/10.1590/S1517-97022015400100301

Noy, C. (2008). Sampling knowledge: The hermeneutics of snowball sampling in qualitative research. International Journal of Social Research Methodology, 11(4), 327-344. https://doi.org/10.1080/13645570701401305

Ong, A. (2007). Neoliberalism as a mobile technology. Transactions of the Institute of British Geographers, 32(1), 3-8. https://doi.org/10.1111/j.1475-5661.2007.00234.x

Ozga, J., Seddon, T. y Popkewitz, T. S. (2006). Education research and policy: Steering the knowledgebased economy. World yearbook of education 2006. Londres: Routledge.

Read, J. (2009). A genealogy of homo-economicus: Neoliberalism and the production of subjectivity. Foucault Studies, 6, 25-36. https://doi.org/10.22439/fs.voio.2465

Rose, N. (1999). Powers of freedom. Reframing political thought. Cambridge: Cambridge University Press. https://doi.org/10.1017/CBO9780511488856

Saura, G. (2016). Neoliberalismo como discurso. La evaluación en educación entre tecnologías políticas neoliberales y la nueva filantropía. Educaçao, Sociedade $\S^{\circ}$ Culturas, 47, 11-30.

Scharff, C. (2017). The psychic life of neoliberalism: Mapping the contours of entrepreneurial subjectivity. Theory, Culture \& Society, 33(6), 107-122.

https://doi.org/10.1177/0263276415590164

Shore, C. (2010). Beyond the multiversity: Neoliberalism and the rise of the schizophrenic university. Social Anthropology, 18(1), 15-29. https://doi.org/10.1111/j.14698676.2009.00094.x 
Shore, C. y Wright, S. (2015). Audit culture revisited. Rankings, rantings, and the reassembling of society. Current Anthropology, 56(3), 42 1-444. https://doi.org/10.1086/681534

Slaughter, S. y Leslie, L. L. (2001). Expanding and elaborating the concept of academic capitalism. Organization, 8(2), 154-161. https://doi.org/10.1177/1350508401082003

Springer, S. (2012). Neoliberalism as discourse: Between Foucauldian political economy and Marxian poststructuralism. Critical Discourse Studies, 9(2), 133-147. https://doi.org/10.1080/17405904.2012.656375

Sugrue, C. (2015). Unmasking school leadership: A longitudinal life history of school leaders. Dordrecht: Springer. https://doi.org/10.1007/978-94-017-9433-6

Tight, M. (2019). The neoliberal turn in higher education. Higher Education Quarterly, 73(3), 273284. https://doi.org/10.1111/hequ.12197

Torres, C. y Schugurensky, D. (2002). The political economy of higher education in the era of neoliberal globalization: Latin America in comparative perspective. Higher Education, 43, 429-455. https://doi.org/10.1023/A:1015292413037

Van Dijk, T. A. (2008). Discourse and power. Houndmills: Palgrave-Macmillan. https://doi.org/10.1007/978-1-137-07299-3

Williamson, B. (2017). Big data in education: The digital future of learning, policy and practice. Londres: SAGE.

\section{Breve CV de los autores}

\section{Geo Saura}

Profesor Ayudante Doctor (2018-2023) en Política Educativa de la Universidad de Granada (Andalucía, España). Investigador Postdoctoral en la Universitat Autònoma de Barcelona (2015-2018) e Investigador Predoctoral (2012-2015) desarrollando su PhD internacional sobre políticas de privatización y neoliberalismo, entre la Universidad de Granada y University of Roehampton, que recibió el Premio Extraordinario a la mejor Tesis Doctoral en Ciencias Sociales. Ha desarrollado múltiples estancias y proyectos de investigación con financiación internacional (América Latina, Europa y España). Sus publicaciones son un intento por capturar las reformas globales explorando estructuras, actores, redes y prácticas en la neoliberalización contemporánea de la política educativa. ORCID ID: https://orcid.org/o000-0002-2365-9532.Email: geosaura@ugr.es

\section{Antonio Bolívar}

Catedrático de Universidad de Didáctica y Organización Escolar de la Universidad de Granada (España). Ha participado, como director o miembro de equipo, en más de veinte de Proyectos de investigación. Ha ejercido de evaluador en diversos Agencias y Proyectos, actualmente lo es en la en el Programa Academia de ANECA. Tiene varios centenares de publicaciones, entre sus últimos libros "Políticas actuales de mejora y liderazgo educativo" (2012); y "Una dirección escolar con capacidad de liderazgo pedagógico" (2019).; (con J. Domingo): "La investigación (auto)biográfica en educación". (Barcelona: Octaedro, 2019). Editor (con J. Domingo) de la Revista Profesorado. Revista de Currículum y Formación del Profesorado (http://recyt.fecyt.es/index.php/profesorado). ORCID ID: http://orcid.org/o000-0001-8818-5799. Email: abolivar@ugr.es 